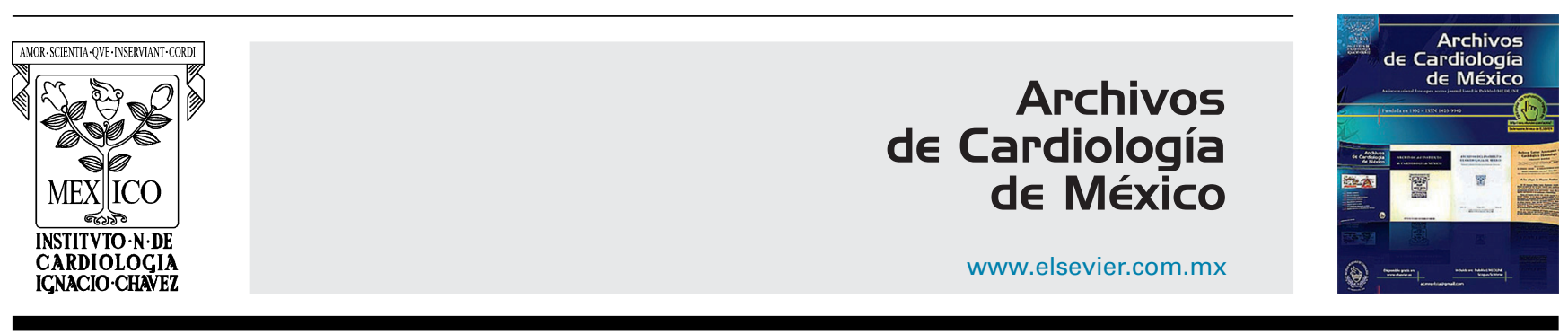

INVESTIGACIÓN CLÍNICA

\title{
Diseño de un registro de fibrilación auricular y riesgo embólico en México: CARMEN-AF
}

\section{Jesús A. González-Hermosillo ${ }^{a}$, Manlio F. Márquez ${ }^{a, *}$ y Salvador Ocampo-Peña ${ }^{b}$, en nombre del Comité Científico del Registro CARMEN-AF}

\author{
a Instituto Nacional de Cardiología «lgnacio Chávez», Ciudad de México, México \\ b Hospital Ángeles de Lindavista, Ciudad de México, México
}

Recibido el 8 de junio de 2016; aceptado el 9 de noviembre de 2016

\author{
PALABRAS CLAVE \\ Anticoagulantes \\ orales; \\ Fibrilación auricular; \\ Registro; \\ Tromboprofilaxis, \\ México
}

\begin{abstract}
Resumen
Objetivo: La fibrilación auricular (FA) es una de las arritmias más comunes, y su prevalencia aumenta con la edad. Se asocia con alto riesgo de embolia cerebral. La prevención de dichas tromboembolias se realiza mediante anticoagulantes orales, que en nuestro país parecen estar subutilizados. El Registro CARMEN-AF tiene como objetivo primario determinar cuál es el estado actual de la tromboprofilaxis de la FA no valvular en México. Como objetivo secundario pretende conocer la morbimortalidad asociada a la FA no valvular en por lo menos un año de seguimiento. Métodos: El Registro CARMEN-AF es un estudio observacional, longitudinal, multicéntrico y nacional sobre el empleo de los anticoagulantes orales en pacientes con FA no-valvular que pretende la inclusión de pacientes mayores de 18 años de edad diagnosticados con FA novalvular durante los últimos 6 meses y con al menos un factor de riesgo para desarrollar una tromboembolia de acuerdo con la escala de $\mathrm{CHA}_{2} \mathrm{DS}_{2}$-Vasc. Serán recolectados datos demográficos y clínicos en las visitas clínicas habituales a lo largo de un seguimiento de 2 años. El reclutamiento comenzó el 19 de septiembre de 2014 y se prevé la inclusión del último paciente el 18 de septiembre de 2016. Se estima la inclusión de 1,200 pacientes dada la incidencia de FA reportada a nivel mundial y tomando en consideración la población mexicana total.

Conclusiones: El registro de FA y riesgo embólico en México (CARMEN-AF) permitirá conocer el estado actual de la tromboprofilaxis en pacientes con FA novalvular y permitirá obtener una panorámica del cumplimiento de las guías nacionales e internacionales de práctica clínica en esta materia.

(c) 2016 Instituto Nacional de Cardiología Ignacio Chávez. Publicado por Masson Doyma México S.A. Este es un artículo Open Access bajo la licencia CC BY-NC-ND (http://creativecommons. org/licenses/by-nc-nd/4.0/).
\end{abstract}

\footnotetext{
* Autor para correspondencia. Instituto Nacional de Cardiología «lgnacio Chávez». Juan Badiano 1. Sección XVI. Tlalpan. C.P. 14080, Ciudad de México. Teléfono: +5255 55133740 .

Correo electrónico: manlio.marquez@gmail.com (M.F. Márquez).

$\diamond$ La totalidad de participantes en el estudio aparecen en el Anexo 1.
} 


\section{KEYWORDS}

Oral anticoagulants; Atrial fibrillation; Registry; Thromboprophylaxis, Mexico

\section{Design of an atrial fibrillation and embolic risk registry in Mexico: CARMEN-AF}

\begin{abstract}
Objective: Atrial fibrillation (AF) is one of the most common arrhythmias, and its prevalence increase with age. It is associated with high risk of stroke. The prevention of such thromboembolism is done with oral anticoagulants, which in our country seem to be underused. CARMEN-AF registry aims primarily to determine the current status of thromboprophylaxis of non-valvular AF in Mexico. A secondary objective is to know the morbidity and mortality associated with non-valvular AF in at least one year of follow-up.

Methods: CARMEN-AF registry is an observational, longitudinal, multicenter, and national survey about the use of oral anticoagulants in patients with non-valvular AF. Patients 18 years old or older, diagnosed with AF during the last 6 months, and with at least one risk factor of thromboembolism based in the $\mathrm{CHA}_{2} \mathrm{DS}_{2}$-Vasc score are being selected. Demographic and clinical data will be collected during the visits to their usual clinic with a follow-up of 2 years. The recruitment began on September 19, 2014, and the inclusion of the last patient is expected on September 18, 2016. According to the reported incidence of AF globally and taking into account the total Mexican population, the inclusion of 1,200 patients is estimated.

Conclusions: The Atrial Fibrillation and Embolic Risk Registry (CARMEN-AF) will reveal the current status of thromboprophylaxis in patients with non-valvular AF, and will allow to get an overview of the national and international clinical practice guidelines accomplishment in this area.

(C) 2016 Instituto Nacional de Cardiología Ignacio Chávez. Published by Masson Doyma México S.A. This is an open access article under the CC BY-NC-ND license (http://creativecommons. org/licenses/by-nc-nd/4.0/).
\end{abstract}

\section{Introducción}

La fibrilación auricular (FA) es la arritmia sostenida más frecuente en la práctica clínica ${ }^{1,2}$. Afecta alrededor del $1-2 \%$ de la población general ${ }^{3}$, su prevalencia aumenta con la edad y tiene múltiples causas, entre las que se encuentran la insuficiencia cardíaca, la diabetes mellitus tipo 2 y la hipertensión arterial sistémica ${ }^{4}$. La FA es un importante factor de riesgo de presentar un evento vascular cerebral (EVC), ya que es responsable del $25 \%$ de los EVC isquémicas y del $50 \%$ de las cardioembólicas. Los pacientes con FA tienen una incidencia de isquemia cerebral del $7 \%$ anual $^{5}$. Además, está demostrado que la FA incrementa la mortalidad hospitalaria ${ }^{6}$, aumenta el número de hospitalizaciones y disminuye la calidad de vida ${ }^{7}$. Así, la FA representa un problema de salud pública con un elevado costo económico asociado $^{8,9}$.

Uno de los objetivos principales del tratamiento de la FA es la prevención de las tromboembolias mediante fármacos anticoagulantes orales (ACO). Las guías terapéuticas de la $\mathrm{FA}^{5,10}$ ofrecen recomendaciones sobre la idoneidad de un paciente para recibir tratamiento con $\mathrm{ACO}$ según las distintas escalas de estratificación de riesgo de EVC $\left(\mathrm{CHADS}_{2}\right.$ y $\left.\mathrm{CHA}_{2} \mathrm{DS}_{2}-\mathrm{Vasc}\right)^{11}$ y de sangrado (HAS-BLED) ${ }^{12}$. La guía de práctica clínica mexicana sigue estas mismas recomendaciones, aunque no hace énfasis en los beneficios de los ACO en los pacientes más longevos o con riesgo elevado, los cuales se suelen tratar con antiagregantes plaquetarios ${ }^{13}$.

Tanto los ACO directos (ACOD) como los antagonistas de la vitamina K (AVK) han mostrado ser efectivos para evitar los EVC en pacientes con $\mathrm{FA}^{14,15}$. Sin embargo, la adherencia a los AVK es muy variable, con una tasa de abandono del tratamiento al primer año que puede llegar hasta un $25 \%$, y múltiples interacciones medicamentosas. El incumplimiento con la medicación está relacionado con una mala percepción por parte del paciente de la necesidad de tomar los medicamentos o por el temor a los efectos adversos $^{16}$. Para mejorar la adherencia es necesaria más información, para que los pacientes conozcan el riesgo que representa su enfermedad, lo consideren seriamente y se involucren en la prevención esencial y en las conductas de tratamiento.

El objetivo principal del Registro CARMEN-AF (FibrilaCión Auricular y Riesgo de troMboembolias EN México) es conocer el estado actual de la tromboprofilaxis en la FA novalvular en México con el fin de identificar las características del empleo actual de los ACO en México. En forma secundaria, se evaluará en un seguimiento mínimo de un año la morbimortalidad asociada con esta arritmia y la eficacia y seguridad en la tromboprofilaxis de la FA con el uso de los nuevos y específicos ACO.

\section{Métodos}

El registro CARMEN-AF es un estudio observacional, longitudinal, multicéntrico y nacional de la FA novalvular. El registro CARMEN-AF se plantea como el primer registro acerca del tratamiento del riesgo tromboembólico en FA novalvular en México que contará con representación nacional. Se planea la participación de las principales instituciones de salud, tanto públicas como privadas, de cada entidad federativa para garantizar que el registro sea representativo de la República Mexicana. Se busca contar con una amplia distribución geográfica para comparar la calidad de la atención entre las distintas zonas geográficas del país. 
El período de seguimiento de cada paciente será por un mínimo de 2 años, con evaluaciones periódicas cada 3 meses. La información se registrará en un formato de informe de caso electrónico (Anexo 2; CRF por las siglas en inglés de case report form) almacenado en una base de datos centralizada en la que se incluirán todos los datos necesarios para la realización del estudio. Las variables de evaluación del estudio serán: a) demográficas; b) factores de riesgo asociados; c) del diagnóstico y características de la FA, así como sus manifestaciones clínicas; d) resultados de pruebas de laboratorio y exámenes de gabinete, y e) variables clínicas de desenlaces importantes (morbimortalidad). El registro de los datos se obtendrá de la práctica clínica habitual, siendo las fuentes de información los propios investigadores participantes y los datos de la historia clínica del paciente en el momento de la inclusión en el estudio.

\section{Criterios de inclusión y exclusión}

Los candidatos para el estudio son aquellos pacientes de cualquier género, mayores de 18 años de edad que tienen documentado mediante un ECG de 12 derivaciones, tira de ritmo, monitoreo Holter ECG o electrogramas auriculares de un marcapasos al menos un episodio de FA en los últimos 6 meses. Todos los pacientes con diagnóstico de FA en la visita basal deben ser elegibles para recibir profilaxis antitrombótica con ACO, y deben tener al menos un factor de riesgo para tromboembolia de acuerdo a la escala $\mathrm{CHA}_{2} \mathrm{DS}_{2}$-Vasc. En el caso del género femenino, debe estar acompañado de algún otro de los factores de riesgo, ya que de lo contrario no se codificará para el puntaje de riesgo medido por $\mathrm{CHA}_{2} \mathrm{DS}_{2}$-Vasc. No se incluirá en el estudio a los pacientes con FA de causa transitoria, con FA de aparición en el postoperatorio inmediato o mediato (3 meses) de cirugía cardíaca, con enfermedad terminal, con incapacidad mental para tomar el anticoagulante o con incapacidad para cumplir con las visitas de seguimiento, pacientes programados para la ablación de venas pulmonares, ni mujeres embarazadas o en periodo de lactancia (tabla 1).

\section{Análisis de los datos}

Para el análisis de los datos se utilizarán estadísticos descriptivos y diferenciales de acuerdo a la observación obtenida. Para las variables continuas se obtendrán como estadísticos descriptivos: media, desviación estándar (DE), error estándar (EE), intervalo de confianza 95\% (IC 95\%) medio, mínimo, percentil 25 (P25), mediana o percentil 50 (P50), percentil 75 (P75) y máximo, número de pacientes (n) y número de datos perdidos. Para las variables categóricas se obtendrán el porcentaje (\%) respecto al total y al número de pacientes (n) de cada categoría. Los datos perdidos se tratarán como una nueva categoría.

Se examinarán mediante la prueba t de Student diferencias entre variables continuas con distribución normal. La prueba del rango de suma de Wilcoxon se utilizará cuando variables continuas hayan fallado en pruebas de normalidad. Para analizar variables categóricas se utilizará ji-cuadrado mediante la prueba de exacta de Fisher o de corrección de Yates. Una prueba de 2 colas con un valor de $p<0.05$ se considerará estadísticamente significativa. Se utilizará el
Tabla 1 Criterios de inclusión y exclusión del estudio

Criterios de inclusión
Sexo: masculino o femenino
Edad: $\geq 18$ años
Paciente con diagnóstico de FA crónica (permanente)
documentada en cuando menos 2 trazos
electrocardiográficos tomados en días diferentes en los
últimos 6 meses. Los trazos pueden ser ECG de
12 derivaciones, tira de ritmo de monitor
electrocardiográfico o Holter de $24 \mathrm{~h}$
Paciente con diagnóstico de FA paroxística/persistente
documentada durante al menos 30 s mediante ECG de
12 derivaciones, tira de ritmo de monitor
electrocardiográfico, Holter de $24 \mathrm{~h}$ o electrogramas
auriculares de un marcapasos. Se requiere que haya
ocurrido y documentado al menos un episodio en los
últimos 6 meses
Paciente con cuando menos un factor de riesgo para
tromboembolias (EVC o fuera del SNC) de acuerdo a la
escala CHA DS ${ }_{2}$-Vasc
Criterios de exclusión
FA de causa transitoria (tirotoxicosis, intoxicación por
alcohol, fase aguda de IM, pericarditis, miocarditis,
electrocución, embolismo pulmonar u otra enfermedad
pulmonar, desorden hidroelectrolítico o metabólico, etc.)
FA de aparición en el postoperatorio inmediato o mediato
(3 meses) de cirugía cardíaca
Enfermedad terminal
Incapacidad mental para tomar el anticoagulante
Incapacidad para cumplir con las visitas de seguimiento
Sujeto ya programado para la ablación de venas
pulmonares
Mujeres embarazadas o en periodo de lactancia
Contraindicación para la anticoagulación oral (anemia,
trombocitopenia, daño hepático o renal graves, etc.)

análisis de regresión logística para seleccionar predictores independientes en aquellas variables en las que a través de un análisis de regresión univariado se haya obtenido un valor de $p<0.01$. Se utilizarán curvas de supervivencia de KaplanMeier y el modelo de riesgo proporcional de Cox para ajustar el análisis de supervivencia. Se considerará como estadísticamente significativa una $p<0.05$.

\section{Resultados}

La fase de reclutamiento comenzó el 19 de septiembre de 2014 y terminará el 18 de septiembre de 2016. Se estima la participación de un total de 1,200 pacientes. Se realizará un primer análisis preliminar con los primeros 1,000 pacientes. En septiembre de 2016 se realizará el análisis de corte transversal de todos los pacientes incluidos (se calculan 1,200 pacientes), y posteriormente se realizarán análisis de seguimiento a uno y 2 años.

Las sociedades participantes y que dan apoyo a este Registro son, por orden alfabético: Asociación Mexicana para la Prevención de la Aterosclerosis y sus Complicaciones (AMPAC), Asociación Nacional de Cardiólogos al 
Servicio de los Trabajadores del Estado (ANCISSSTE), Asociación Nacional de Cardiólogos del Centro Medico La Raza A.C., Asociación Nacional de Cardiólogos de México A.C. (ANCAM), Instituto Nacional de Cardiología «lgnacio Chávez» (INCICH), Sociedad Mexicana de Cardiología A.C. (SMC) y
Sociedad Mexicana de Electrofisiología y Estimulación Cardíaca (SOMEEC).

Actualmente se cuenta con 51 instituciones participantes confirmadas hasta el momento para incluir pacientes en el registro (tabla 2).

Tabla 2 Instituciones participantes

\begin{tabular}{|c|c|c|}
\hline Estado & Ciudad & Centro \\
\hline Aguascalientes & Aguascalientes & Cardiológica Aguascalientes \\
\hline \multirow[t]{4}{*}{ Baja California } & Tijuana & Hospital Ángeles de Tijuana \\
\hline & & Hospital Regional No. 1, IMSS \\
\hline & & ISSSTECALI \\
\hline & & Plaza Medical \\
\hline Baja California Sur & La Paz & Hospital General La Paz, ISSSTE \\
\hline \multirow[t]{2}{*}{ Campeche } & Campeche & Hospital General de Especialidades INDESALUD \\
\hline & & Hospital General de Zona No. 1, IMSS \\
\hline Chiapas & Tuxtla Gutiérrez & Policlínica de especialidades \\
\hline Chihuahua & Chihuahua & CIMA Chihuahua \\
\hline \multirow[t]{8}{*}{ Ciudad de México } & Ciudad de México & Centro Médico Nacional «20 de noviembre», ISSSTE \\
\hline & & Centro Médico Nacional «La Raza», IMSS \\
\hline & & Hospital ABC \\
\hline & & Hospital Central de Alta Especialidad Norte, PEMEX \\
\hline & & Hospital de Cardiología, Centro Médico Nacional «Siglo XXI», IMSS \\
\hline & & Hospital General de México \\
\hline & & Instituto Nacional de Cardiología «lgnacio Chávez» \\
\hline & & Instituto Nacional de Ciencias Médicas y Nutrición «Salvador Zubirán» \\
\hline Coahuila & Torreón & Unidad Médica de Alta Especialidad No. 71, IMSS \\
\hline Colima & Colima & Centro Médico «Puerta de Hierro» \\
\hline Durango & Durango & Hospital General de Durango \\
\hline Estado de México & Toluca & ISSEMYM Toluca \\
\hline \multirow[t]{2}{*}{ Guanajuato } & Guanajuato & Unidad Médica de Alta Especialidad No. T-1, IMSS \\
\hline & León & Hospital Ángeles de León \\
\hline \multirow[t]{2}{*}{ Guerrero } & Acapulco & Hospital General de Acapulco \\
\hline & Iguala & Hospital General de Iguala \\
\hline \multirow[t]{2}{*}{ Hidalgo } & Pachuca & Consultorio privado, Dr. Lechuga \\
\hline & & Hospital General de Pachuca \\
\hline \multirow[t]{2}{*}{ Jalisco } & Guadalajara & Centro Médico Nacional de Occidente, IMSS \\
\hline & & Hospital Civil de Guadalajara \\
\hline Michoacán & Uruapan & Hospital General de Uruapan, SSA \\
\hline Morelos & Cuernavaca & Instituto Mexicano de Trasplantes \\
\hline Nayarit & Tepic & Consultorio privado, Dr. Varela \\
\hline Nuevo León & Monterrey & Hospital San José \\
\hline Oaxaca & Oaxaca & Clínica Molina \\
\hline \multirow[t]{2}{*}{ Puebla } & Puebla & Hospital Ángeles de Puebla \\
\hline & & Hospital General del Sur de Puebla, SSA \\
\hline Querétaro & Querétaro & Instituto Corazón de Querétaro \\
\hline Quintana Roo & Cancún & Hospital General de Zona No. 3, IMSS \\
\hline \multirow[t]{2}{*}{ San Luis Potosí } & San Luis Potosí & Hospital Ángeles de San Luis Potosí \\
\hline & & Hospital Central «Morones Prieto» \\
\hline \multirow[t]{2}{*}{ Sinaloa } & Culiacán & Hospital Ángeles de Culiacán \\
\hline & & Hospital Civil de Culiacán \\
\hline Sonora & Ciudad Obregón & Centro Médico Nacional del Noroeste, IMSS \\
\hline Tabasco & Villahermosa & Hospital Regional de Alta Especialidad Villahermosa, SSA \\
\hline \multirow[t]{2}{*}{ Tamaulipas } & Tampico & Hospital Ángeles de Tampico \\
\hline & & Hospital Regional Tampico, PEMEX \\
\hline Veracruz & Veracruz & Unidad Médica de Alta Especialidad No. 14, IMSS \\
\hline Yucatán & Mérida & Star Médica Mérida \\
\hline \multirow[t]{2}{*}{ Zacatecas } & Zacatecas & Hospital General No. 26, ISSSTE \\
\hline & & Hospital San Agustín \\
\hline
\end{tabular}




\section{Discusión}

Al igual que sucede en el resto del mundo, en América Latina el incremento en la esperanza de vida de la población aumenta la incidencia de EVC isquémicas, y se teme que aumente su mortalidad en los próximos años ${ }^{17,18}$. Entre 2003 y 2005 el EVC ocurrió en el 6.9\% de la población mexicana, y fue la principal causa de mortalidad en las zonas urbanas, afectando al 14.9\% de dicha población ${ }^{19}$. En 2010, el $0.9 \%$ de los egresos del sector público sanitario mexicano fueron registros de EVC aguda, de los cuales 20,298 (43.9\%) fueron EVC isquémica ${ }^{20}$. Por otro lado, la epidemiología de la FA en América Latina es poco conocida. En 2007 se estimó que el $60.7 \%$ de las arritmias en México eran debidas a FA, de las cuales el $65.7 \%$ eran de tipo no valvular.

Una parte importante del tratamiento de la FA es la prevención de las tromboembolias. La terapia anticoagulante es altamente efectiva para reducir el riesgo de EVC en estos pacientes ${ }^{21}$; no obstante, cada paciente debe ser valorado de manera individual para determinar los riesgos y los beneficios de la terapia antitrombótica. Las guías actuales de práctica clínica recomiendan administrar los anticoagulantes de acuerdo a las distintas escalas de estratificación de riesgo, para poder tomar las decisiones del tratamiento de acuerdo al puntaje obtenido ${ }^{22}$. Así, las guías canadienses aconsejan el uso de la escala $\mathrm{CHADS}_{2}$ para valorar el riesgo de EVC y la escala HAS-BLED para el riesgo de hemorragia, y el uso de los ACOD (dabigatrán, rivaroxabán o apixabán) frente a los $\mathrm{AVK}^{23}$. La guía estadounidense recomienda el uso de antitrombóticos como norma general, excepto en los casos contraindicados ${ }^{24}$. Finalmente, las guías europeas recomiendan el uso de la escala $\mathrm{CHA}_{2} \mathrm{DS}_{2}$ Vasc y HAS-BLED para medir los riesgos de EVC y sangrado, respectivamente, y el uso de ACOD frente a aspirina y $\mathrm{AVK}^{10}$. Todas estas recomendaciones están basadas en los resultados obtenidos en estudios de fase III, como ARISTOTLE, RE-LY o ROCKET AF ${ }^{25-28}$, que han mostrado ventajas en la seguridad, eficacia y manejo de los ACOD frente a los $\mathrm{AVK}^{29}$. Las recomendaciones de la guía de práctica clínica mexicana también favorecen el tratamiento con $\mathrm{ACO}^{5}$.

A pesar de las recomendaciones existentes y de las evidencias a favor de los tratamientos con ACO, se estima que solo entre el 15 y el $79 \%$ de los pacientes reciben este tratamiento en forma adecuada ${ }^{30}$. Los AVK tienen una farmacocinética compleja que obliga a una vigilancia continua del tiempo de protrombina, frecuentes ajustes de la dosis, con un elevado riesgo de hemorragia, además de que tienen múltiples interacciones con otros fármacos y alimentos ${ }^{30}$. La facilidad para la administración, la poca interacción con otros fármacos y la menor necesidad de vigilancia de los ACOD hacen esperable un incremento en el uso del tratamiento anticoagulante en los pacientes con $\mathrm{FA}^{31}$.

La adherencia a los AVK es muy variable. El estudio ATRIA documentó un abandono del $25 \%$ en el primer año en los pacientes tratados con $\mathrm{AVK}^{32}$, mientras que el estudio INRANGE documentó que el $92 \%$ de los pacientes omitieron una dosis y el $36 \%$ omitieron más del $20 \%$ del tratamiento ${ }^{33}$. Esta falta de adherencia es una de las principales causas de los eventos embólicos. Por otra parte, no existe información sobre la adherencia a los ACOD, aunque se espera que esta sea mayor que con los AVK por su dosificación fija, facilidad de administración, innecesario monitoreo, mayor eficacia y seguridad. Para obtener un buen seguimiento de la medicación es necesario disminuir la asimetría de la información, pero también es importante cumplir con las recomendaciones de las guías internacionales de práctica clínica.

\section{Conclusión}

El estudio CARMEN-AF permitirá obtener información del estado actual de la tromboprofilaxis en pacientes con FA no valvular en México. Se analizarán las estrategias de tratamiento anticoagulante en un total de 1,200 pacientes y se evaluará su eficacia. Además, se podrá valorar el grado de cumplimiento de las guías de práctica clínica, lo que permitirá elaborar una estrategia educativa para mejorar la adherencia a este tipo de tratamiento.

\section{Responsabilidades éticas}

Protección de personas y animales. Los autores declaran que para esta investigación no se han realizado experimentos en seres humanos ni en animales.

Confidencialidad de los datos. Los autores declaran que en este artículo no aparecen datos de pacientes.

Derecho a la privacidad y consentimiento informado. Los autores declaran que en este artículo no aparecen datos de pacientes.

\section{Financiamiento}

Este proyecto tiene el apoyo irrestricto Bayer, BoehringerIngelheim y Pfizer; el cual está destinado a fines académicos.

\section{Conflicto de intereses}

El Registro cuenta con un patrocinio irrestricto de la industria farmacéutica sin que esta intervenga en la elaboración y diseño del mismo. La recolección de los datos se llevará a cabo por una agencia especializada, independiente de la industria patrocinadora. El análisis de los datos correrá a cargo de los miembros del Comité Científico, quien será también el encargado de coordinar las publicaciones que se puedan generar de dichos análisis.

Ninguno de los autores que participan en esta investigación tiene conflicto de interés de ningún tipo con Bayer, Boehringer-Ingelheim, Pfizer, Medicaweb o con alguna de las sociedades médicas participantes.

\section{Agradecimientos}

A la Sociedad Mexicana de Cardiología, por otorgar el aval a este proyecto. A Medicaweb, por el soporte técnico brindado para el desarrollo de este Registro nacional.

\section{Anexo 1. Participantes en el estudio}

Comité científico:

Dr. J. Antonio González-Hermosillo (coordinador nacional); Dr. Manlio F. Márquez Murillo (presidente); Dr. Salvador 
Ocampo Peña (secretario); Dr. Guillermo Ceballos; Dr. Alejandro Cordero; Dr. Marcelo Jiménez; Dr. Armando García; Dr. Efraín Gaxiola; Dr. Jorge Gómez; Dr. Enrique Martínez; Dr. Luis Molina; Dr. Gerardo Rodríguez-Diez; Dr. Humberto Rodríguez; Dr. Juan Pablo Benítez.

Comité financiero:

Dr. Luis Molina; Dr. Bernardo Quintana; Dr. Rubén Yza Villanueva, AMPAC; Dr. Enrique Velázquez, ANCCMN «La Raza»; Dr. José Manuel Enciso, ANCAM; Dr. Rogelio Robledo,
ANCISSSTE; Dr. Jorge Gómez (temporalmente), SMC; Dr. Susano Lara, SOMEEC.

Representantes de la industria:

Dra. Alejandra Meaney, Bayer; Dra. Yuriria Valle, Boehringer-Ingelheim; Dra. M. Eugenia Pastrana, Pfizer.

Organización de investigación por contrato (CRO por las siglas en inglés de contract research organization): Medicaweb, S.A. de C.v.

\section{Anexo 2. CRF del estudio}

Institución:

Variables Demográficas

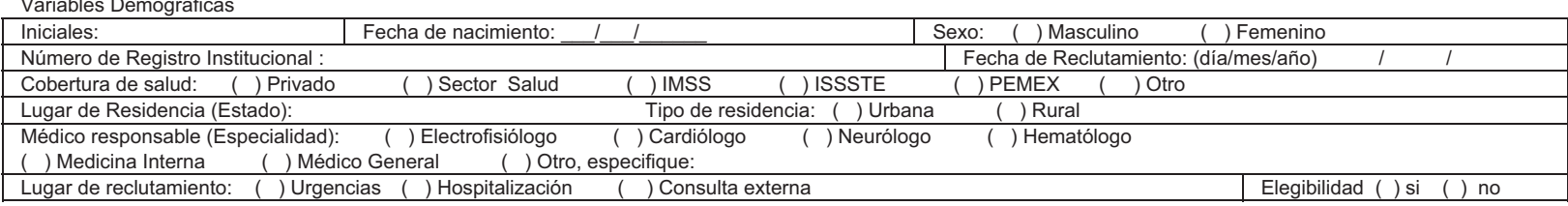

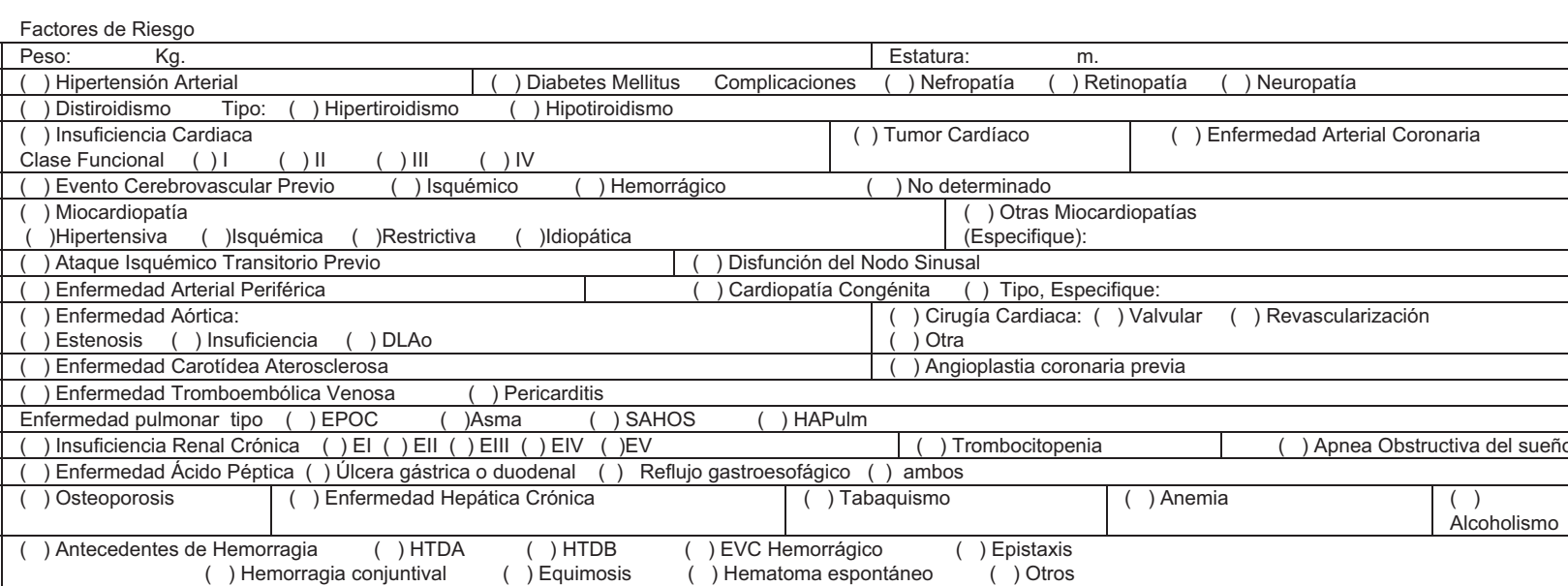

Variables Diagnósticas de Fibrilación Auricular

Método diagnóstico de fibrilación auricular (seleccione uno) :

$\begin{array}{ll}\text { ( ) Electrocardiograma de } 12 \text { derivaciones } & \text { ( ) Tira de Ritmo }\end{array}$

( ) Monitoreo electrocardiográfico de 24 horas ( ) Monitorización de marcapaso permanente

Tipo: ( )Paroxística ( )Persistente ( )Permanente

Manifestaciones Clínicas

Manifestaciones Clínicas
\begin{tabular}{|l|l|l|}
\hline ( ) Dolor Torácico & ( ) Palpitaciones & $($ Presíncope \\
\hline ( ) Mareos & ( ) Disnea & $($ Síncope \\
\hline ( ) Descompensación de insuficiencia cardiaca & ( ) Fatiga & $($ ) Edema \\
\hline ( ) Desencadenamiento de angina & ( ) Asintomático & \\
\hline
\end{tabular}

( ) Desencadenamiento de angina

Electrocardiograma

\begin{tabular}{|c|c|}
\hline $\begin{array}{lll}\text { Ritmo: } & (\text { )FA } & \text { ( ) Sinusal } \\
\end{array}$ & \\
\hline Duración QRS: & Duración onda $\mathrm{P}^{*}$ : \\
\hline ( ) Bloqueo de rama derecha & Intervalo PR*: \\
\hline ( ) Bloqueo de rama izquierda & Intervalo QT*: \\
\hline Frecuencia cardíaca & Frecuencia ventricular media* \\
\hline
\end{tabular}

Ecocardiografía Básica

\begin{tabular}{|l}
\hline \multicolumn{2}{|l|}{$\begin{array}{c}\text { Aurícula derecha : Dimensiones vista apical de } 4 \text { cámaras (Fin de la sístole ventricular): } \\
\text { Longitudinal }\end{array}$} \\
\hline Aurícula izquierda: Dimensiones vista apical de 4 cámaras (Fin de la sístole ventricular): \\
Longitudinal
\end{tabular}


Anticoagulantes

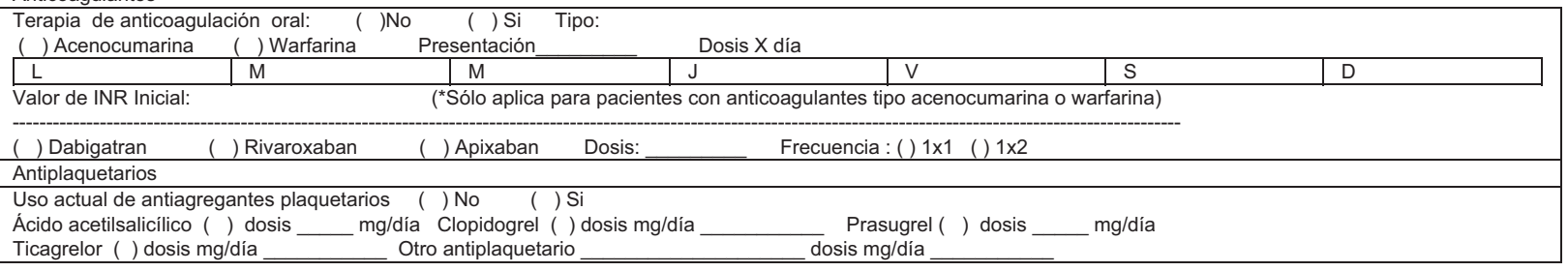

ProcedimientosPrevios

( ) Cardioversión Eléctrica $\quad$ Ritmo sinusal final: ( ) No ( )Si

( ) Aislamiento de Venas Pulmonares $\quad$ Ritmo sinusal final: ( ) No ( ) $\mathrm{Si}$

( ) Cirugíade laberinto (maze) $\quad$ Ritmo sinusal final: ( )No ( ) Si

( ) Ablación del nodo AV + Implante de Marcapaso

\begin{tabular}{lllll}
\hline ( ) Implante de Marcapaso: & Disfunción del nodo sinusal ( ） & Disfunción del Nodo auriculo ventricular ( ) & FA lenta ( )
\end{tabular}

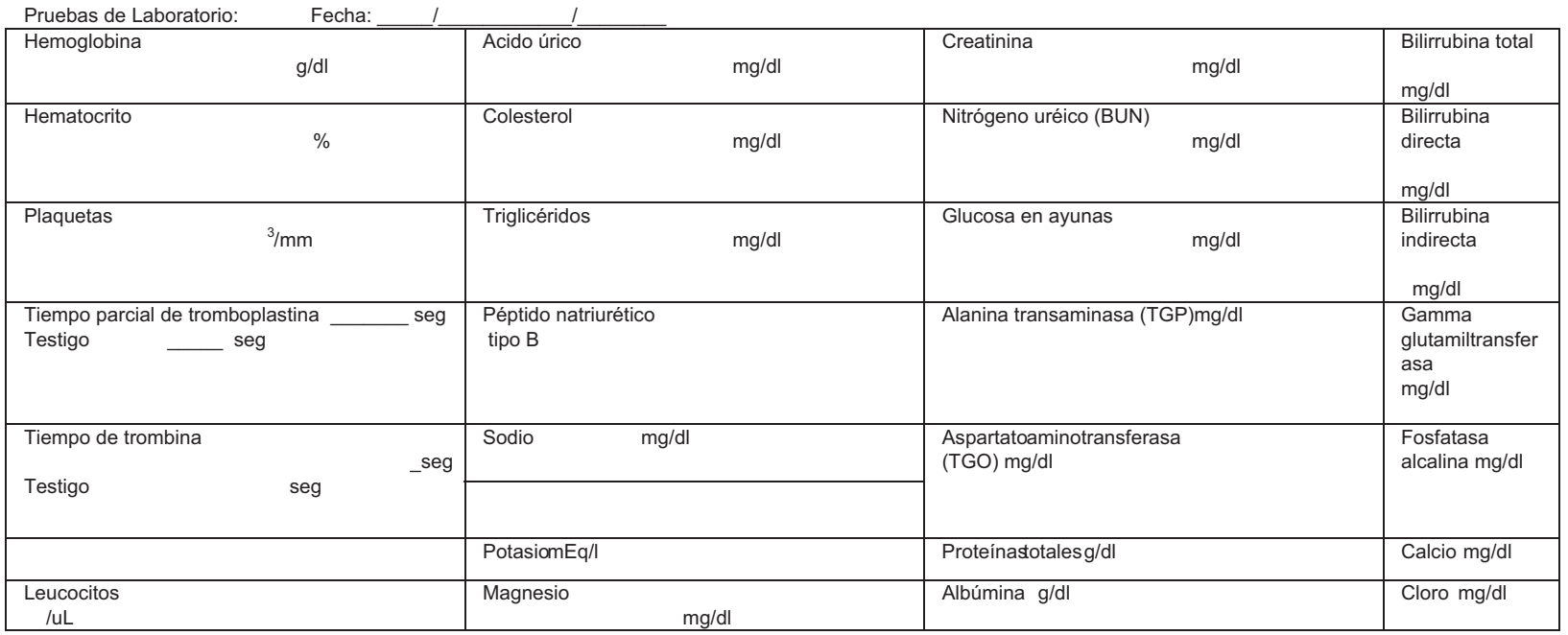

Llenado por:

\section{Bibliografía}

1. Kakkar AK, Mueller I, Bassand JP, et al. International longitudinal registry of patients with atrial fibrillation at risk of stroke: Global Anticoagulant Registry in the FIELD (GARFIELD). Am Heart J. 2012;163:13-9.

2. Iturralde-Torres P, Lara-Vaca S, Cordero-Cabra A, et al. Diseño de un registro multicéntrico para evaluar control de ritmo contra control de la frecuencia en fibrilación auricular: Registro Mexicano de Fibrilación Auricular (ReMeFA). Arch Cardiol Mex. 2011;81:13-7.

3. Go AS, Hylek EM, Phillips KA, et al. Prevalence of diagnosed atrial fibrillation in adults: National implications for rhythm management and stroke prevention: The AnTicoagulation and Risk Factors in Atrial Fibrillation (ATRIA) Study. JAMA. 2001;285:2370-5.

4. Benjamin EJ, Levy D, Vaziri SM, et al. Independent risk factors for atrial fibrillation in a population-based cohort. The Framingham Heart Study. JAMA. 1994;271:840-4.

5. Castaño-Guerra RJ, Franco-Vergara BC, Baca-López FM, et al. Guía de práctica clínica. Diagnóstico y tratamiento de la fibrilación auricular. Rev Med Inst Mex Seguro Soc. 2012;50:213-31.

6. González-Pacheco H, Márquez MF, Arias-Mendoza A, et al. Clinical features and in-hospital mortality associated with different types of atrial fibrillation in patients with acute coronary syndrome with and without ST elevation. J Cardiol. 2015;66:148-54.

7. Rivero-Ayerza M, Scholte op Reimer W, Theuns DAMJ, et al. New-onset atrial fibrillation is an independent predictor of in-hospital mortality in hospitalized heart failure patients:
Results of the EuroHeart Failure Survey. Eur Heart J. 2008;29: 1618-24.

8. Go AS, Mozaffarian D, Roger VL, et al. Heart disease and stroke statistics - 2013 update: A report from the American Heart Association. Circulation. 2013;127:e6-245.

9. Fuster V, Rydén LE, Cannom DS, et al. ACC/AHA/ESC 2006 guidelines for the management of patients with atrial fibrillation: Full text: A report of the American College of Cardiology/American Heart Association Task Force on practice guidelines and the European Society of Cardiology Committee for Practice Guidelines (Writing Committee to Revise the 2001 guidelines for the management of patients with atrial fibrillation) developed in collaboration with the European Heart Rhythm Association and the Heart Rhythm Society. Europace. 2006;8:651-745.

10. Camm J, Kirchhof P, Lip GYH, et al. Guidelines for the management of atrial fibrillation. Eur Heart J. 2010;31:2369-429.

11. Olesen JB, Torp-Pedersen C, Hansen ML, et al. The value of the CHA2DS2-VASC score for refining stroke risk stratification in patients with atrial fibrillation with a CHADS2 score 0-1: A nationwide cohort study. Thromb Haemost. 2012;107:1172-9.

12. Pisters R, Lane DA, Nieuwlaat R, et al. A novel user-friendly score (HAS-BLED) to assess 1-year risk of major bleeding in patients with atrial fibrillation: The Euro Heart Survey. Chest. 2010;138:1093-100.

13. Martínez-Flores JE. Reflexiones sobre la guía clínica en fibrilación auricular. Rev Med Inst Mex Seguro Soc. 2012;50: 117-22.

14. Hart RG, Pearce LA, Aguilar MI. Meta-analysis: Antithrombotic therapy to prevent stroke in patients who have nonvalvular atrial fibrillation. Ann Intern Med. 2007;146:857-67. 
15. Laupacis A, Dalen J, Feinberg W. Antithrombotic therapy in atrial fibrillation. Chest. 1995;108:352S-9S.

16. Kimmel SE, Chen Z, Price M, et al. The influence of patient adherence on anticoagulation control with warfarin. Results from the International Normalized Ratio Adherence and Genetics (INRANGE) study. Arch Intern Med. 2007;167:229-35.

17. Hart RG, Benavente O, McBride R, et al. Antithrombotic therapy to prevent stroke in patients with atrial fibrillation: a metaanalysis. Ann Intern Med. 1999;131:492-501.

18. Demographics and Retirement Policy in Brazil, Chile, and Mexico. Global Aging Initiative. Center for Strategic and International Studies. 2009 [consultado 21 Mar 2013]. Disponible en: http://csis.org/files/media/csis/pubs/090324_gai_english.pdf

19. Ferri CP, Acosta D, Guerra M, et al. Socioeconomic factors and all cause and cause-specific mortality among older people in Latin America, India, and China: A population-based cohort study. PLoS Med. 2012;9:e1001179, http://dx.doi.org/10.1371/ journal.pmed.1001179.

20. Chiquete E, Ruiz-Sandoval JL, Murillo-Bonilla LM, et al. Egresos por enfermedad vascular cerebral aguda en instituciones públicas del sector salud de México: Un análisis de 5.3 millones de hospitalizaciones en 2010. Rev Mex Neuroci. 2012;13: 252-8.

21. Lip GYH. Recommendations for thromboprophylaxis in the 2012 focused update of the ESC guidelines on atrial fibrillation: A commentary. J Thromb Haemost. 2013;11:615-26.

22. Camm AJ, Lip GY, De Caterina R, et al. 2012 focused update of the ESC guidelines for the management of atrial fibrillation: An update of the 2010 ESC guidelines for the management of atrial fibrillation-developed with the special contribution of the European Heart Rhythm Association. Europace. 2012; $14: 1385-413$

23. Skanes AC, Healey JS, Cairns JA, et al. Focused 2012 update of the Canadian Cardiovascular Society atrial fibrillation guidelines: Recommendations for stroke prevention and rate/rhythm control. Canadian J Cardiol. 2012;28:125-36.

24. Anderson JL, Halperin JL, Albert NM, et al. Management of patients with atrial fibrillation (compilation of 2006 ACCF/AHA/ESC and 2011 ACCF/AHA/HRS recommendations). A report of the American College of Cardiology/American Heart Association task force on practice guidelines. Circulation. 2013;127:1916-26.

25. Granger CB, Alexander JH, McMurray JJ, et al. Apixaban versus warfarin in patients with atrial fibrillation. N Engl J Med. 2011;365:981-92.

26. Connolly SJ, Ezekowitz MD, Yusuf S, et al. Dabigatran versus warfarin in patients with atrial fibrillation. N Engl J Med. 2009;361:1139-51.

27. Patel MR, Mahaffey KW, Garg J, et al. Rivaroxaban versus warfarin in nonvalvular atrial fibrillation. N Engl J Med. 2011;365:883-91.

28. Tendera M, Syzdót M, Parma Z. ARISTOTLE RE-LYs on the ROCKET. What's new in stroke prevention in patients with atrial fibrillation. Cardiol J. 2012;19:4-10.

29. Buckingham TA, Hatala R. Anticoagulants for atrial fibrillation: why is the treatment rate so low. Clin Cardiol. 2002;25:447-54.

30. Michota F. Transitions of care in anticoagulated patients. J Multidiscip Healthc. 2013;6:215-28.

31. Singer DE, Chang Y, Fang MC, et al. The net clinical benefit of warfarin anticoagulation in atrial fibrillation. Ann Intern Med. 2009;151:297-305.

32. Ferguson C, Inglis SC, Newton PJ, et al. Atrial fibrillation and thromboprophylaxis in heart failure: The need for patientcentered approaches to address adherence. Vasc Health Risk Manag. 2013;9:3-11.

33. Horne R. Patients' beliefs about treatment: The hidden determinant of treatment outcome. J Psychosom Res. 1999;47:491-5. 